\title{
Semi-empirical ( PM3) studies of novel Aminopyridino-1- 4-n- cyclohexa-1,3-diene iron tricarbonyl complexes
}

\author{
Odiaka, T.I, Adejoro, I.A and Akinyele, O.F. \\ Department of Chemistry, University of Ibadan, Ibadan Nigeria. \\ Email address: ajibadejoro@yahoo.com
}

\begin{abstract}
Theoretical studies on the geometries, electronic states, thermodynamic parameters and vibrational frequencies of new aminopyridino-1-4-n- cyclohexa-1,3-diene complexes were carried out. The theoretical ground state geometries, electronic structure, thermodynamic properties and vibrational frequencies were obtaianed using PM3 method. The geometries,electronic states, thermodynamic properties and vibrational frequencies are discussed. The results show that all the complexes studied are thermodnamically stable
\end{abstract}

Keywords: Aminopyridino, thermodynamic parameters, electronic properties, dipole moments, modeling.

\section{INTRODUCTION}

Modeling is an aspect of Computation in Chemistry and a particular molecular system can be modeled with the hope that it could be synthesized in the Laboratory. Although molecular modeling may not be perfect, it has been proved to be excellent at predicting the properties of compounds, including those that can not be determined experimentall. It is an aspect of Chemistry that is spreading and enjoying the patronage of theoretical Chemists due to its predictive ability in studies involving molecular systems. ${ }^{2}$ Various theoretical models have been applied in studying the properties, electronic states and stabilities of various complexes ${ }^{3}$

This paper reveals studies that were carried out on molecular systems involving a number of organometallic complexes shown in Figure 1.The complexes were modeled using Spartan '08 and theoretical investigations were carried out on optimized geometries using semi-empirical PM 3 method. The results show that these complexes are thermodynamically stable with virtually no significant change in geometries of the pyridine ring and the viccinity of the amino substituent. The structures of these complexes are shown in Figure 1

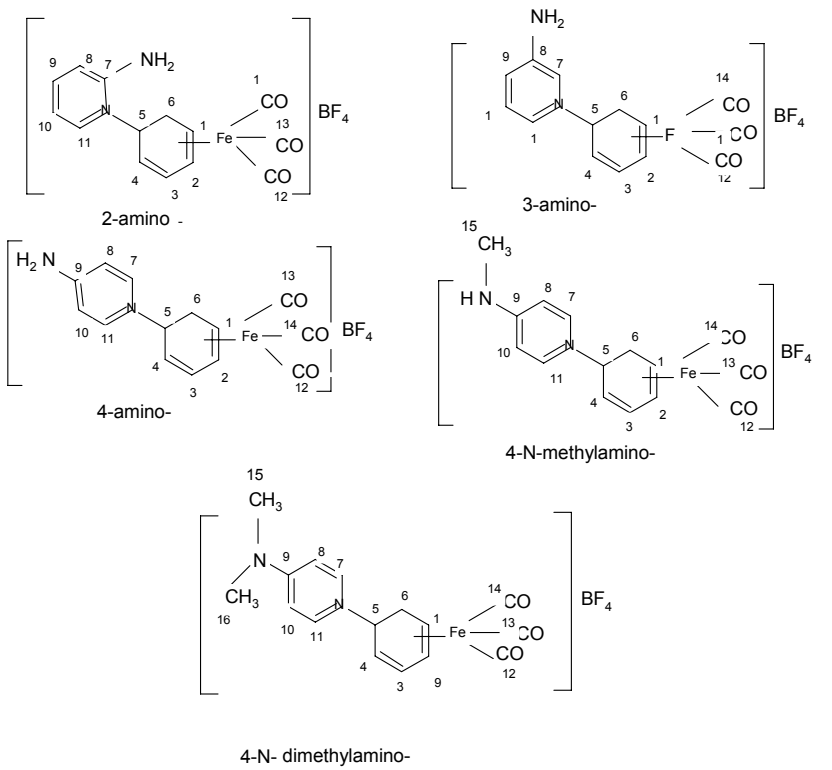

Fig 1: Structure of optimized geometries of novel aminopyridino-1-4-n-cyclohexa-1,3-diene irontricarbonyl complexes 
Am. J. Sci. Ind. Res., 2012, 3(1): 1-13

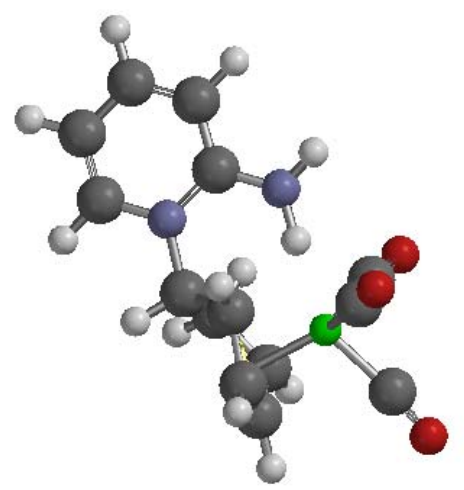

2-aminopyridino-1-4-n-cyclohexa-1,3-dieneirontricarbonyl

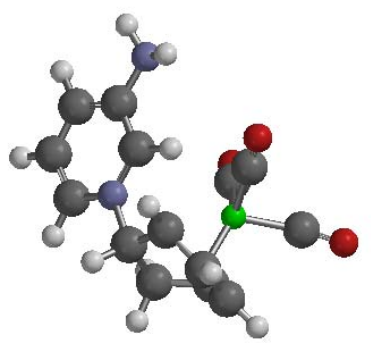

3- aminopyridino-1-4-n-cyclohexa-1,3-dieneirontricarbonyl 
Am. J. Sci. Ind. Res., 2012, 3(1): 1-13

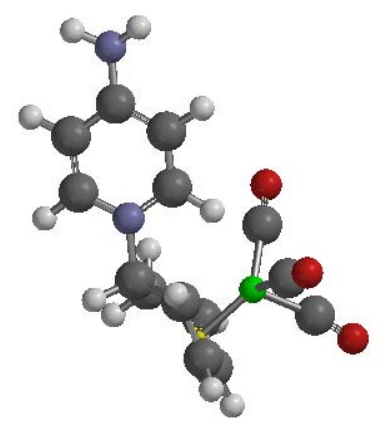

4-aminopyridino-1-4-n-cyclohexa-1,3-dieneirontricarbonyl

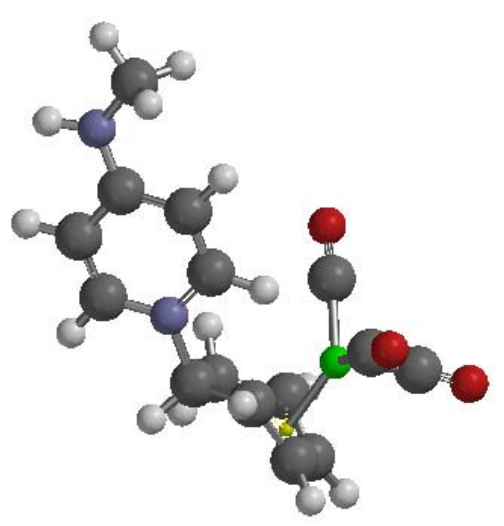

4-N-methylaminopyridino-1-4-n-cyclohexa-1,3-dieneirontricarbonyl

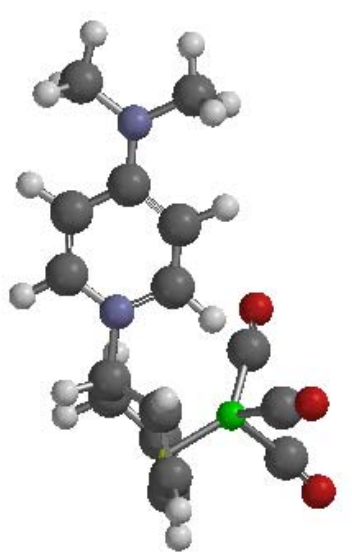

4-N-dimethylaminopyridino-1-4-n-cyclohexa-1,3-dieneirontricarbonyl

Fig 2: Structure of optimized geometries of novel aminopyridino-1-4-n-cyclohexa-1,3-diene irontricarbonyl complexes. (the $\mathrm{BF}_{4}$ is excluded for clearity.) 
Computational Methodology : All the complexes were modeled using Spartan '08. Semi-empirical molecular calculations were carried out on all complexes involved. All the structures were fully optimized.

Electronic states, thermodynamic properties, vibrational frequencies, dipole moments and geometries were carried out using Semi-empirical PM3 method. The optimized geometries of these complexes are shown in Figure 2.

\section{RESULTS AND DISCUSSION}

Geometric parameters : The geometrical parameters were obtained after total optimization of the equilibrum eometries by Semi-empirical PM3. Bond length, bond angles and dihedral angles which contribute to the internal energies were measured and recorded. (See Table 1). The effects of the substituent amino group on the pyridine is clearly noticeable around the adjacent carbon atoms; for instance, the bond length of $\mathrm{N}-\mathrm{C}_{7}$ in the 2aminopyridino complex is $1.398 \AA$ because the amino substituent is attached to $C_{7}, C_{7}-C_{8}$ bond length is also $1.432 \AA$. These values are more than the values obtained for other pyridino substituents and this can be attributed to the electron donor property of the amino group. $\mathrm{C}_{7}-\mathrm{N}_{2}$ is also $1.366 \AA$ which is low in comparison with other $\mathrm{C}-\mathrm{N}$ amino substituents such

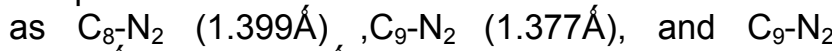
$(1.470 \AA \hat{)})$ and $1.380 \AA$ for $\mathrm{N}$-methyl and $\mathrm{N}$-dimethyl substituents respec- tively. With the amino substituent on $\mathrm{C}_{8}$, the bond length $\mathrm{C}_{7}-\mathrm{C}_{8}$ reduces to $1.412 \AA$ while $C_{8}-C_{9}$ bond length increases to $1.413 \AA$ and the adjacent $\mathrm{C}_{9}-\mathrm{C}_{10}$ bond length reduces to $1.383 \AA$. This is a clear manifestation of the substuent effect on the attached pyridine ring. All the $\mathrm{C}-\mathrm{C}$ single and double bonds are in the range of 1.432-1.529 and 1.368-1.489 $\AA$ respectively. The metal-carbon bond falls within the range $1.730-1.817 \AA$ while all the metal-ligand bond is within 1.697-1.701 $\mathrm{A}$ with little or no significant changes. All the $\mathrm{C}-\mathrm{H}$ bonds fall within $090 \AA$ and $1.122 \AA$. However, the bond and dihedral angles are collected in Table 1.

Electronic properties: It is pertinent to examine the HOMO-LUMO in order to explain the electronic properties of these new organometallics. The HOMO of these complexes possesses a r-bonding character

within sub-units and $\pi$-antibonding character excited state properties between the consecutive subunits. On the other hand, the LUMO possesses a $\pi$-antibonding character within the sub-unit and a $\pi-$ bonding character between the subunits. Experimentally, the HOMO and the LUMO energies were obtained from an empirical formula based on the onset of oxidation-

reduction of peaks measured by cyclic voltametry. Theoretrically, the HOMO and LUMO

energies are calculated using Semi-empirical PM3. These calculations however, do not have solid- state packing effect and aqueous state is not taken into consideration. Although these energy levels may not be accurate, it is possible to use them to obtain information by comparing them with other compounds. The calculated electronic properties (energy band gap,( LUMO-HOMO) of these complexes were found to be within $7.30-7.80 \mathrm{eV}$. The calculated values are shown in Table 2 while the LUMO-HOMO structures are shown in Figure 3. There is a significant change in the values recorded for dipole moments.

Thermodynamic properties and stabilities : The formation of these amino pyridino-1-4- $\eta$-cyclohexa1,3-diene iron tricarbonyl organometallics are spontaneous. For complexes to be thermodynamically stable, it is expected that $\Delta G$ and $\Delta \mathrm{H}$ are negative. The more negative these values are and the more positive $\Delta S$, the more stable would be the amino pyridino complexes. However all calculated free energy and Enthalpy changes are negative while all calculated entropy values are positive, thus confirming that the formation of these complexes is spontaneous. Our calculations reveal that all the complexes are thermodynamically stable as shown in Table 3

Vibrational Frequencies : According to group representation theory in Chemistry, there are $93 \mathrm{~A}$ type vibrational modes for these new aminopyridino organometallics. The vibrational modes changes with the replacement of the hydrogen atom with methyl groups. This vibrational modes increase to 102 when the hydrogen atoms are completely replaced by methyl groups, The vibrational modes rose to 111 . Among these normal modes, the strongest infra-red absorption peaks and their intensities are shown in Table 4 while the simulated IR spectra of these complexes are shown in Figure 4 
Am. J. Sci. Ind. Res., 2012, 3(1): 1-13

Table 1: Geometric parameters (bond distances IÁ, bond angles, dihedral angles in degrees) of the studied aminopyridino-1-4-n- cyclohexa-1,3-diene irontricarbonyl complexes.

(a) Selected bond length:

\begin{tabular}{|c|c|c|c|c|c|}
\hline Bond length / & $2-\mathrm{NH}_{2}$ & $3-\mathrm{NH}_{2}$ & $4-\mathrm{NH}_{2}$ & $4-\mathrm{NH}\left(\mathrm{CH}_{3}\right)$ & $4-\mathrm{N}\left(\mathrm{CH}_{3}\right)_{2}$ \\
\hline $\mathrm{C}_{1}-\mathrm{C}_{2}$ & 1.465 & 1.477 & 1.473 & 1.473 & 1.473 \\
\hline $\mathrm{C}_{2}-\mathrm{C}_{3}$ & 1.405 & 1.396 & 1.398 & 1.398 & 1.398 \\
\hline $\mathrm{C}_{3}-\mathrm{C}_{4}$ & 1.489 & 1.485 & 1.489 & 1.487 & 1.487 \\
\hline $\mathrm{C}_{4}-\mathrm{C}_{5}$ & 1.509 & 1.496 & 1.498 & 1.499 & 1.499 \\
\hline $\mathrm{C}_{5}-\mathrm{C}_{6}$ & 1.539 & 1.539 & 1.540 & 1.540 & 1.540 \\
\hline $\mathrm{C}_{6}-\mathrm{C}_{1}$ & 1.498 & 1.494 & 1.495 & 1.495 & 1.495 \\
\hline $\mathrm{C}_{5}-\mathrm{N}_{1}$ & 1.510 & 1.519 & 1.519 & 1.515 & 1.514 \\
\hline $\mathrm{N}_{1}-\mathrm{C}_{7}$ & 1.398 & 1.366 & 1.378 & 1.378 & 1.379 \\
\hline $\mathrm{C}_{7}-\mathrm{C}_{8}$ & 1.432 & 1.412 & 1.379 & 1.378 & 1.377 \\
\hline $\mathrm{C}_{8}-\mathrm{C}_{9}$ & 1.368 & 1.413 & 1.418 & 1.419 & 1.424 \\
\hline $\mathrm{C}_{9}-\mathrm{C}_{10}$ & 1.416 & 1.383 & 1.420 & 1.424 & 1.426 \\
\hline $\mathrm{C}_{10}-\mathrm{C}_{11}$ & 1.369 & 1.398 & 1.377 & 1.375 & 1.375 \\
\hline $\mathrm{C}_{11}-\mathrm{N}_{1}$ & 1.399 & 1.369 & 1.383 & 1.384 & 1.383 \\
\hline $\mathrm{lig}_{-}-\mathrm{Fe}$ & 1.701 & 1.700 & 1.697 & 1.697 & 1.697 \\
\hline $\mathrm{Fe}-\mathrm{C}_{12}$ & 1.765 & 1.734 & 1.730 & 1.730 & 1.730 \\
\hline $\mathrm{Fe}-\mathrm{C}_{13}$ & 1.815 & 1.799 & 1.798 & 1.797 & 1.797 \\
\hline $\mathrm{Fe}-\mathrm{C}_{14}$ & 1.817 & 1.809 & 1.804 & 1.803 & 1.802 \\
\hline $\mathrm{C}_{12}-\mathrm{O}_{1}$ & 1.154 & 1.159 & 1.160 & 1.160 & 1.160 \\
\hline $\mathrm{C}_{13}-\mathrm{O}_{2}$ & 1.145 & 1.148 & 1.148 & 1.148 & 1.148 \\
\hline $\mathrm{C}_{14}-\mathrm{O}_{3}$ & 1.144 & 1.145 & 1.146 & 1.146 & 1.146 \\
\hline $\mathrm{N}_{2}-\mathrm{H}$ & 0.990 & 0.993 & 0.991 & - & - \\
\hline $\mathrm{C}_{7}-\mathrm{N}_{2}$ & 1.367 & - & - & - & - \\
\hline $\mathrm{C}_{8}-\mathrm{N}_{2}$ & - & 1.399 & - & - & - \\
\hline $\mathrm{C}_{9}-\mathrm{N}_{2}$ & - & - & 1.377 & 1.470 & 1.380 \\
\hline $\mathrm{N}_{2}-\mathrm{C}$ & - & - & - & 1.480 & 1.480 \\
\hline $\mathrm{C}-\mathrm{H}$ & $1.090-1.122$ & $1.091-1.123$ & $1.090-1.124$ & $1.090-1.124$ & $1.090-1.124$ \\
\hline
\end{tabular}

(b) Bond angles in degrees :

\begin{tabular}{|c|c|c|c|c|c|}
\hline Bond angle/ & $2-\mathrm{NH}_{2}$ & 3- $\mathrm{NH}_{2}$ & 4- $\mathrm{NH}_{2}$ & 4-NH($\left(\mathrm{CH}_{3}\right)$ & $4-\mathrm{N}\left(\mathrm{CH}_{3}\right)_{2}$ \\
\hline $\mathrm{C}_{12}$-Fe-lig & 133.62 & 133.04 & 132.22 & 132.17 & 132.13 \\
\hline $\mathrm{C}_{13}$-Fe-lig & 120.41 & 119.15 & 119.61 & 119.65 & 119.62 \\
\hline $\mathrm{C}_{14}-\mathrm{Fe}-\mathrm{lig}$ & 109.29 & 111.27 & 111.91 & 111.95 & 112.02 \\
\hline $\mathrm{Fe}-\mathrm{C}_{12}-\mathrm{O}_{1}$ & 169.89 & 170.41 & 171.52 & 171.65 & 171.61 \\
\hline $\mathrm{Fe}-\mathrm{C}_{13}-\mathrm{O}_{2}$ & 174.23 & 174.86 & 174.92 & 174.97 & 174.82 \\
\hline $\mathrm{Fe}-\mathrm{C}_{14}-\mathrm{O}_{3}$ & 170.13 & 170.75 & 170.98 & 170.94 & 171.07 \\
\hline $\mathrm{C}_{5}-\mathrm{N}_{1}-\mathrm{C}_{11}$ & 118.51 & 117.46 & 119.09 & 119.25 & 119.42 \\
\hline $\mathrm{C}_{5}-\mathrm{N}_{1}-\mathrm{C}_{7}$ & 122.18 & 122.05 & 121.03 & 120.97 & 121.12 \\
\hline $\mathrm{N}_{1}-\mathrm{C}_{7}-\mathrm{N}_{2}$ & 119.19 & - & - & $\begin{array}{c}- \\
\end{array}$ & $\begin{array}{c}- \\
\end{array}$ \\
\hline $\mathrm{C}_{7}-\mathrm{N}_{1}-\mathrm{C}_{11}$ & 119.19 & 120.47 & 119.89 & 119.78 & 119.89 \\
\hline $\mathrm{C}_{7}-\mathrm{C}_{8}-\mathrm{N}_{2}$ & & 119.73 & - & - & - \\
\hline $\mathrm{C}_{8}-\mathrm{C}_{9}-\mathrm{N}_{2}$ & - & - & 120.97 & 122.67 & 121.70 \\
\hline $\mathrm{C}_{9}-\mathrm{N}_{2}-\mathrm{C}_{15}$ & - & - & - & 122.83 & 118.26 \\
\hline
\end{tabular}


Am. J. Sci. Ind. Res., 2012, 3(1): 1-13

(c) Dihedral angles in degrees:

\begin{tabular}{|c|c|c|c|c|c|}
\hline Dihedral angles / & $2-\mathrm{NH}_{2}$ & $3-\mathrm{NH}_{2}$ & $4-\mathrm{NH}_{2}$ & $4-\mathrm{NH}\left(\mathrm{CH}_{3}\right)$ & $4-\mathrm{N}\left(\mathrm{CH}_{3}\right)_{2}$ \\
\hline $\mathrm{C}_{4}$-lig-Fe- $\mathrm{C}_{13}$ & 51.20 & 55.48 & 52.85 & 52.57 & 52.51 \\
\hline $\mathrm{C}_{3}$-lig-Fe-C 14 & 83.80 & 91.81 & 90.61 & 90.47 & 90.57 \\
\hline $\mathrm{C}_{2}$-lig-Fe- $\mathrm{C}_{12}$ & 92.23 & 105.30 & 105.93 & 106.02 & 106.14 \\
\hline $\mathrm{N}_{2}-\mathrm{C}_{7}-\mathrm{N}_{1}-\mathrm{C}_{5}$ & 0.86 & - & - & - & - \\
\hline $\mathrm{C}_{5}-\mathrm{N}_{1}-\mathrm{C}_{7}-\mathrm{N}_{2}$ & 0.25 & 3.16 & 0.38 & 0.20 & 0.23 \\
\hline $\mathrm{N}_{2}-\mathrm{C}_{8}-\mathrm{C} 7-\mathrm{N}_{1}$ & - & -175.60 & - & - & - \\
\hline $\mathrm{N}_{2}-\mathrm{C}_{9}-\mathrm{C}_{8}-\mathrm{C}_{7}$ & - & - & -176.03 & -179.77 & -179.80 \\
\hline $\mathrm{C}_{8}-\mathrm{C}_{9}-\mathrm{N}_{2}-\mathrm{C}_{15}$ & - & - & - & -10.34 & -0.08 \\
\hline
\end{tabular}

Table 2: Dipole moments, HOMO and LUMO energies, Energy band gaps ( Eg ) for simulated aminopyridino-1-4-ncyclohexa-1,3-diene irontricarbonyl complexes.

\begin{tabular}{|l|c|c|c|c|}
\hline X-aminopyridino & $\begin{array}{l}\text { Dipole moments / } \\
\text { debit }\end{array}$ & $\begin{array}{l}\text { HOMO energy / } \\
\text { eve }\end{array}$ & $\begin{array}{l}\text { LUMO energy / } \\
\text { eve }\end{array}$ & Band gaps / eve \\
\hline 2-amino- & 2.19 & -12.14 & -4.34 & -7.80 \\
\hline 3-amino- & 4,39 & -12.13 & -4.83 & -7.30 \\
\hline 4-amino- & 6.06 & -12.02 & -4.67 & -7.35 \\
\hline 4-Nmethylamino- & 4.55 & -11.97 & -4.55 & -7.42 \\
\hline 4-N-dimethylamino- & 3.23 & -11.92 & -4.43 & -7.49 \\
\hline
\end{tabular}

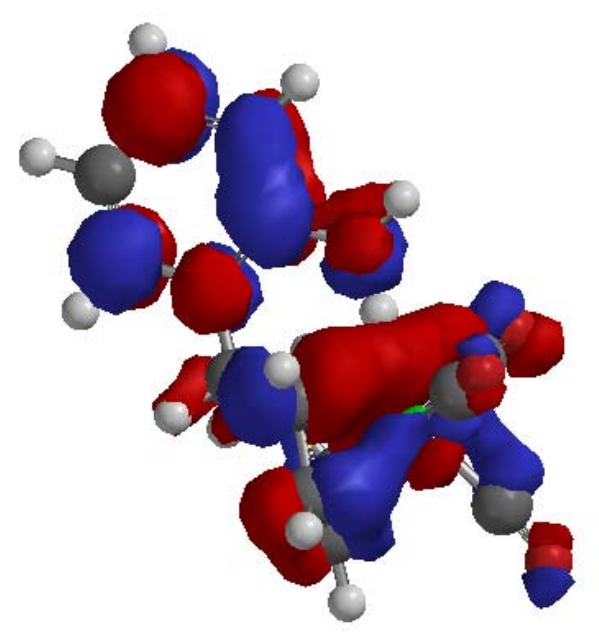

2-aminopyridino-1-4-n-cyclohexa-1,3-dieneirontricarbonyl 
Am. J. Sci. Ind. Res., 2012, 3(1): 1-13

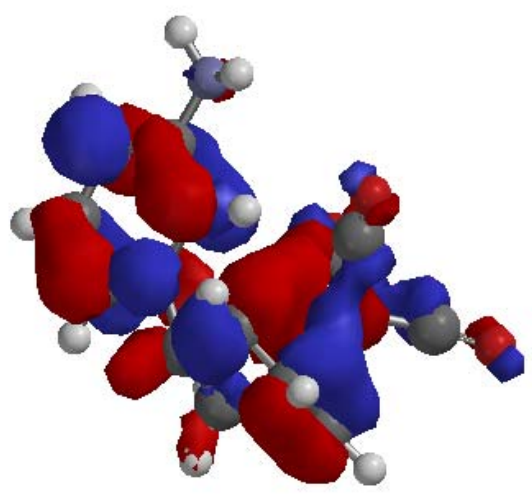

3-aminopyridino-1-4-n-cyclohexa-1,3-dieneirontricarbonyl

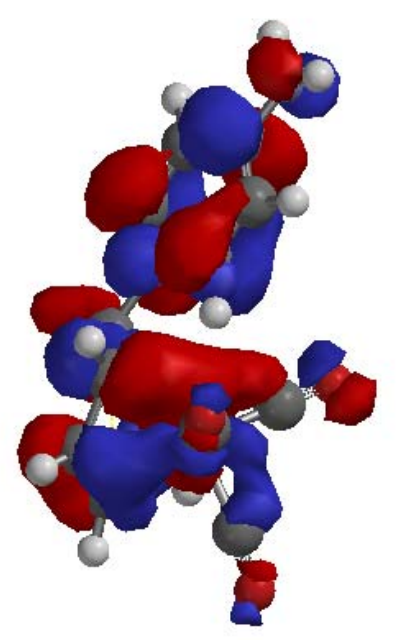

4-aminopyridino-1-4-n-cyclohexa-1,3-dieneirontricarbonyl 
Am. J. Sci. Ind. Res., 2012, 3(1): 1-13

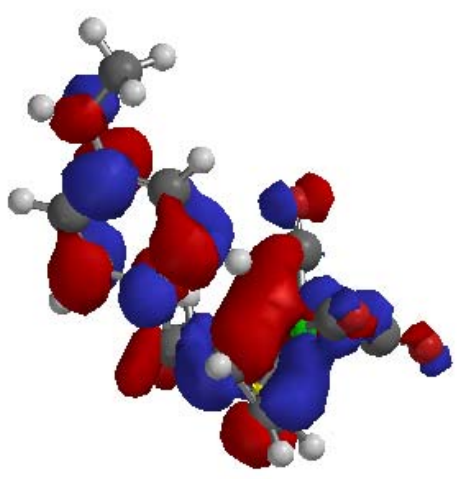

4-N-methylaminopyridino-1-4-n-cyclohexa-1,3-dieneirontricarbonyl

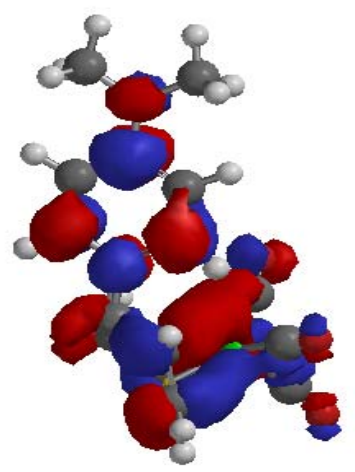

4-N-dimethylaminopyridino-1-4-n-cyclohexa-1,3-dieneirontricarbonyl

Fig 3: HOMO - LUMO Energy diagrams for optimized geometries of aminopyridino-1-4-n-cyclohexa-1,3-diene irontricarbonyl complexes. 
Am. J. Sci. Ind. Res., 2012, 3(1): 1-13

Table 3: Thermodynamic parameters for simulated aminopyridino-1-4- $\eta$ - cyclohexa-1,3-diene irontricarbonyl complexes at $298.15 \mathrm{~K}$.

\begin{tabular}{|l|c|c|c|c|}
\hline X-aminopyridino & Heat of formation/ kJmol & Free energy /kJmol & $\begin{array}{l}\text { Entropy } \\
\mathrm{Jmol}^{-1} \mathrm{~K}^{-1}\end{array}$ & \multicolumn{1}{|c|}{$\begin{array}{l}\text { Enthalpy } \\
\mathrm{kJmol}^{-1}\end{array}$} \\
\hline $2-\mathrm{NH}_{2}$ & -823.56 & -299.46 & 561.27 & -132.11 \\
\hline $3-\mathrm{NH}_{2}$ & -824.92 & -294.32 & 556.66 & -125.37 \\
\hline $4-\mathrm{NH}_{2}$ & -827.87 & -313.01 & 577.85 & -140.72 \\
\hline $4-\mathrm{NH}\left(\mathrm{CH}_{3}\right)$ & -843.48 & -253.70 & 612.03 & -71.22 \\
\hline $4-\mathrm{N}\left(\mathrm{CH}_{3}\right)_{2}$ & -844.13 & -188.50 & 648.50 & 4.86 \\
\hline
\end{tabular}

Table 4: Vibrational frquencies showing absorption bands and intensities for simulated aminopyridino-1,4- $\eta$ cyclohexa-1,3-diene irontricarbonyl complexes.

\begin{tabular}{|c|c|c|c|}
\hline Structure & Vibrating bond & Infra-red band $/ \mathrm{cm}^{-1}$ & Intensity \\
\hline \multirow{8}{*}{$2-\mathrm{NH}_{2}$} & $\mathrm{C}_{5}-\mathrm{N}_{1}$ & 1474 & 2721 \\
\hline & $\mathrm{C}_{7}-\mathrm{N}_{2}$ & 1585 & 1721 \\
\hline & $\mathrm{C}_{8}-\mathrm{C}_{9}$ & 1691 & 17658 \\
\hline & $\mathrm{C}_{10}-\mathrm{C}_{11}$ & 1784 & 1582 \\
\hline & $\begin{array}{l}\mathrm{N}_{2}-\mathrm{H}, \\
\mathrm{C} \equiv \mathrm{O}\end{array}$ & 2129 & 2246 \\
\hline & $\mathrm{C} \equiv \mathrm{O}$ & 2184 & 5951 \\
\hline & $\mathrm{C} \equiv \mathrm{O}$ & 2192 & 3670 \\
\hline & $\mathrm{N}_{2}-\mathrm{H}$ & 2307 & 1750 \\
\hline \multirow[t]{5}{*}{ 3- $\mathrm{NH}_{2}$} & $\mathrm{C}_{11}-\mathrm{N}_{1}$ & 1330 & 3015 \\
\hline & $\mathrm{C}_{8}-\mathrm{C}_{9}$ & 1738 & 1978 \\
\hline & $\mathrm{C} \equiv \mathrm{O}$ & 2106 & 1661 \\
\hline & $\mathrm{C} \equiv \mathrm{O}$ & 2171 & 1217 \\
\hline & $C \equiv O$ & 2241 & 1078 \\
\hline \multirow[t]{8}{*}{$4-\mathrm{NH}_{2}$} & $\mathrm{C}_{11}-\mathrm{N}_{1}$ & 1357 & 1616 \\
\hline & $\mathrm{C}_{10}-\mathrm{C}_{11}$ & 1561 & 2060 \\
\hline & $\mathrm{C}_{8}-\mathrm{C}_{9}$ & 1682 & 10119 \\
\hline & $\mathrm{C}_{7}-\mathrm{C}_{8}$ & 1735 & 1857 \\
\hline & $\mathrm{C} \equiv \mathrm{O}$ & 2103 & 1728 \\
\hline & $C \equiv O$ & 2169 & 1310 \\
\hline & $\mathrm{C} \equiv \mathrm{O}$ & 2239 & 1204 \\
\hline & $\mathrm{C}_{7}-\mathrm{H}$ & 2754 & 1219 \\
\hline \multirow[t]{8}{*}{$4-\mathrm{NH}\left(\mathrm{CH}_{3}\right)$} & $C_{10}-C_{11}$ & 1568 & 2322 \\
\hline & $\mathrm{C}_{9}-\mathrm{N}_{2}$ & 1626 & 1117 \\
\hline & $\mathrm{C}_{9}-\mathrm{C}_{10}$ & 1676 & 11151 \\
\hline & $\mathrm{C}_{7}-\mathrm{C}_{8}$ & 1734 & 1771 \\
\hline & $\mathrm{C} \equiv \mathrm{O}$ & 2102 & 1739 \\
\hline & $\mathrm{C} \equiv \mathrm{O}$ & 2168 & 1323 \\
\hline & $\mathrm{C} \equiv \mathrm{O}$ & 2238 & 1208 \\
\hline & $\mathrm{C}_{7}-\mathrm{H}$ & 2756 & 1310 \\
\hline \multirow[t]{8}{*}{$4-\mathrm{N}\left(\mathrm{CH}_{3}\right)_{2}$} & $\mathrm{C}_{11}-\mathrm{N}_{1}$ & 1360 & 1567 \\
\hline & $\mathrm{C}_{10}-\mathrm{C}_{11}$ & 1571 & 1990 \\
\hline & $\mathrm{C}_{9}-\mathrm{C}_{8}$ & 1663 & 12085 \\
\hline & $\mathrm{C}_{7}-\mathrm{C}_{8}$ & 1734 & 1835 \\
\hline & $\mathrm{C} \equiv \mathrm{O}$ & 2101 & 1742 \\
\hline & $\mathrm{C} \equiv \mathrm{O}$ & 2167 & 1329 \\
\hline & $\mathrm{C} \equiv \mathrm{O}$ & 2238 & 1210 \\
\hline & $\mathrm{C}_{7}-\mathrm{H}$ & 2763 & 1278 \\
\hline
\end{tabular}


Am. J. Sci. Ind. Res., 2012, 3(1): 1-13

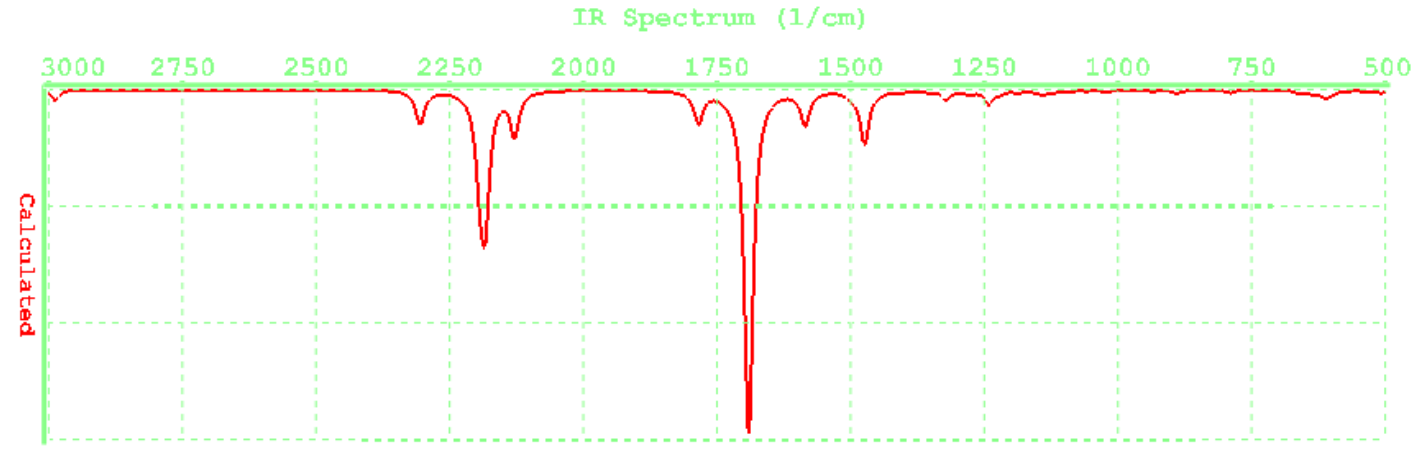

2-aminopyridino-1-4-n-cyclohexa-1,3-dieneirontricarbonyl

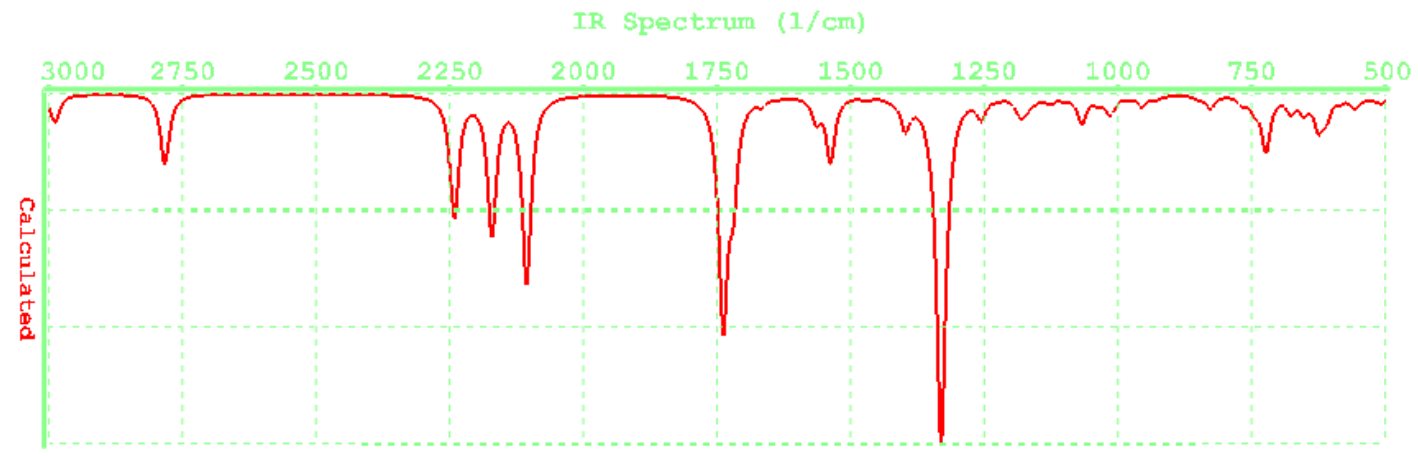

3-aminopyridino-1-4-n-cyclohexa-1,3-dieneirontricarbonyl 
Am. J. Sci. Ind. Res., 2012, 3(1): 1-13

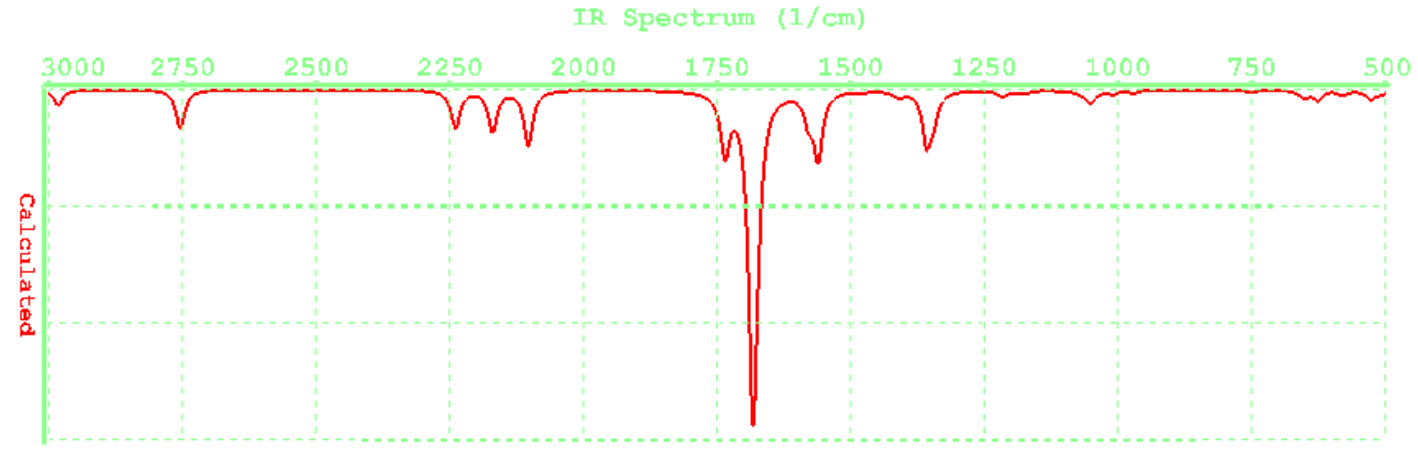

4-aminopyridino-1-4-n-cyclohexa-1,3-dieneirontricarbonyl

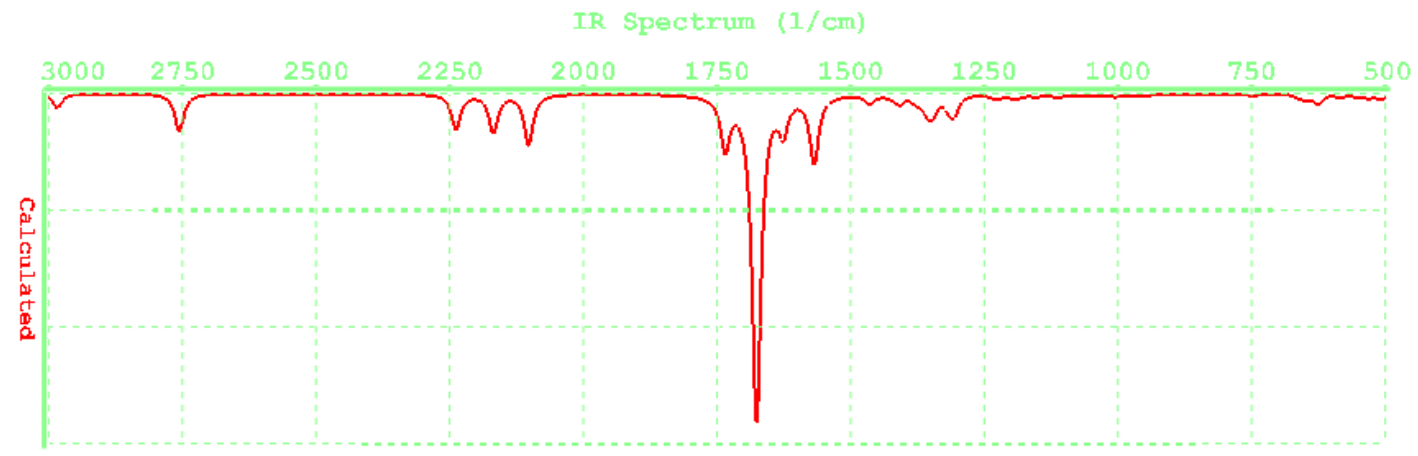

4-N-methylaminopyridino-1-4-n-cyclohexa-1,3-dieneirontricarbonyl 
Am. J. Sci. Ind. Res., 2012, 3(1): 1-13

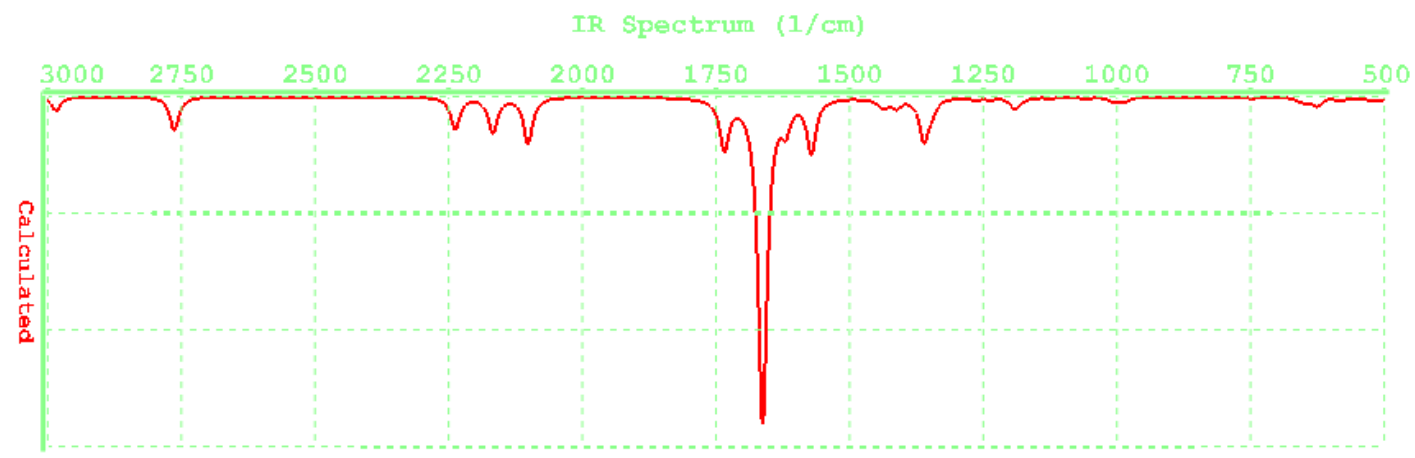

4-N-dimethylaminopyridino-1-4-n-cyclohexa-1,3-dieneirontricarbonyl

Fig 4: Infra-red spectra showing vibrational frequencies and intensities for aminopyridino-1-4-n-cyclohexa-1,3-diene irontricarbonyl complexes.

\section{CONCLUSION}

The C1 symmetry point group resulting from the nucleophilic addition of aminopyridines and

their substituted derivatives to the 1,5-ncyclohexadienyl iron tricarbonyl complex to form new cationic aminopyridino-1-4-n-cyclohexa-1,3-diene iron tricarbonyl complexes were calculated using Semi-empirical PM3. The properties investigated include optimized geometries, dipole moments, electronic states, thermodynamic parameters and vibrational frequencies. All the complexes are thermodynamically stable. The effect of the substituent amino group on the pyridine is demonstrated by the calculated energy band gap of the order of $7.30-7.80 \mathrm{eV}$.( Table 2 ). It is amazing that the use of computation has given us the opportunity to take a critical look at these novel compounds at the molecular level to produce

results which are otherwise inaccessible by conventional laboratory experiments. There are however no experimental or theoretical data regarding these novel Organometallics. Our result may therefore form the basis for reference for further theoretical and experimental work.

ACKNOWLEDGEMENT We are grateful to the University of Ibadan for providing enabling

Environment for this research work.

\section{REFERENCES}

1. David C, Young.(2001) Computational ChemistryA pratical Guide for applying real Word problems. Wiley-Interscience. ISBN : 0-471-333689.

2. Xiao, J.L and Geng, S.T (2009). Theoretical studies of the functionalized derivatives of fullerene $\mathrm{C}_{24} \mathrm{H}_{24}$ by attaching a variety of chemical groups. $\mathrm{J}$. Mol. Struct. Theochem.893, 26-30

3. Zgou, H, Hamidi, M, Lere-Porte, J, Serein-Spirau, F and Bouachrine, M. (2008). Structural and Electronic Properties of New Materials Based on Thiophene and Phenylene. Acta Phys-Chim. Sin. 24(1):37-40

4. Bouzakaraoui, S, Bouzzine, S.M, Bouachrine, M and Hamidi, M, Theoretical Study on the Structure 
and properties of Polythiophine. ( 2005 ) Journal of Molecular. Structure. Theochem. 725 : 39.

5. Bouzzine, S.M, Bouzakraoui, S, Bouachrine, M, Hamidi, M and Hamdaoui. (2004).Structure and Electronic properties of new materials based on Thiophene and Phenylene. Physics Chemistry News $19: 110$.

6. Warren J. Henre (2003).A guide to molecular mechanics and molecular orbital calculations in Spartan. Wavefuntion Inc. ISBN 1-890661-18-X.

7. Odiaka, T.I and Kane-Maguire, L.A.P, Turgoose $S$ and Williams P.A (1981).Kinetics of Nucleophilic Attack on Co-ordinated Organic moieties. Significance of amine basicity in determining the nucleophilicities of pyridines and anilines towards (1-5-dienyl) $\mathrm{Fe}\left(\mathrm{CO}_{3}\right)$ Iron. Journal of Organometallic Chemistry 188: C5 - C9.

8. Odiaka, T.I and Kane-Maguire, L.A.P, Turgoose S and Williams P.A (1981).Kinetics of Nucleophilic Attack on Co-ordinated Organic moieties. Addition of anilines to tricarbonyl (1-5-dienyl) Iron cations. Journal of Chemical Society. Dalton Trans. 2489 2495.

9. Spartan '08V1.11. Wavefunction Inc. Japan.

10. Warren J. Henre, Jianguo $\mathrm{Yu}$, and Philip F. Klunzinger ( 1997) A guide to molecular mechanics and molecular orbital calculations in Spartan. Wavefuntion Inc. ISBN 1-890661-04-X 